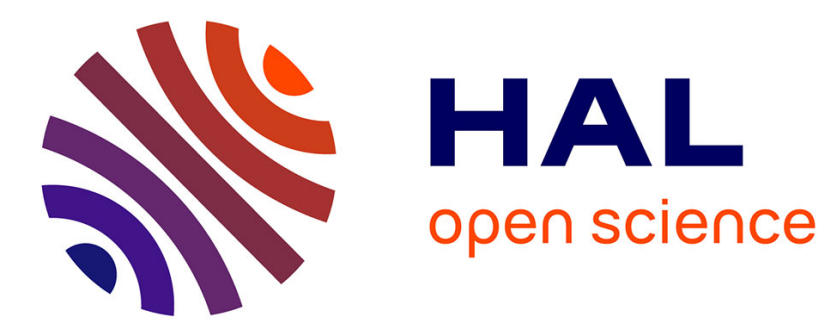

\title{
Occupancy statistics arising from weighted particle rearrangements
}

\author{
Thierry Huillet
}

\section{To cite this version:}

Thierry Huillet. Occupancy statistics arising from weighted particle rearrangements. Journal of Physics A: Mathematical and Theoretical, 2007, 40, pp.9179-9200. hal-00120316

\section{HAL Id: hal-00120316 https://hal.science/hal-00120316}

Submitted on 14 Dec 2006

HAL is a multi-disciplinary open access archive for the deposit and dissemination of scientific research documents, whether they are published or not. The documents may come from teaching and research institutions in France or abroad, or from public or private research centers.
L'archive ouverte pluridisciplinaire HAL, est destinée au dépôt et à la diffusion de documents scientifiques de niveau recherche, publiés ou non, émanant des établissements d'enseignement et de recherche français ou étrangers, des laboratoires publics ou privés. 


\title{
Occupancy statistics arising from weighted particle rearrangements
}

\author{
Thierry Huillet \\ Laboratoire de Physique Théorique et Modélisation, \\ CNRS-UMR 8089 et Université de Cergy-Pontoise, \\ 2 Avenue Adolphe Chauvin, 95302, Cergy-Pontoise, FRANCE \\ E-mail: Thierry.Huillet@u-cergy.fr
}

December 13, 2006

\begin{abstract}
The box-occupancy distributions arising from weighted rearrangements of a particle system are investigated. In the grand-canonical ensemble, they are characterized by determinantal joint probability generating functions. For doubly non-negative weight matrices, fractional occupancy statistics, generalizing Fermi-Dirac and Bose-Einstein statistics, can be defined. A spatially extended version of these balls-in-boxes problems is investigated.
\end{abstract}

Keywords: occupancy problems, urn models, interaction, Boltzmann weight matrices, balls in boxes, MacMahon master theorem, permanents, correlation functions.

AMS Classification 2000: 60C05, 60E05, 05Axx, 82Bxx, 60-02.

PACS Numbers: 02.50.Ey, 02.50.-r, 05.30.Pr, 05.40.-a, 02.10.Yn

\section{Introduction and outline}

The purpose of this work is to study a class of combinatorial balls-in-boxes models as a random allocation scheme of particles. Although the urn model under study is formally in the spirit of the ones found for example in Charalambides, [6], Johnson and Kotz, [16], or Kolchin, [17], books, it is not covered by these manuscripts. Another unrelated balls-in-boxes process of similar flavour was recently revisited in [15], developing some equilibrium aspects of the zeta-urn model first introduced in [4]. In some sense made precise later, the model we 
shall deal with here is concerned with random box filling of an interacting particle system derived from weighted permutations of its constitutive items.

We now describe our model in some detail. Suppose we are given a total amount of $k \geq 1$ labeled particles (items) of $n$ different types (or colors), with $n \geq 2$. Let $k_{m}, m=1, . ., n$ be the number of type- $m$ particles in some initial configuration, with $\left|\mathbf{k}_{n}\right|:=k_{1}+. .+k_{n}=k$ and $\mathbf{k}_{n}=\left(k_{m} ; m=1, . ., n\right)$. Place initially these $k$ particles in boxes and more specifically, place the $k_{m}$ type- $m$ particles in box number $m, m=1, . ., n$, respectively. Suppose the energy required to move a particle from box $m$ to box $m^{\prime}$ is $-\log W_{m, m^{\prime}}$ where $W_{m, m^{\prime}}$ is the $m \times m^{\prime}$ entry of some non-negative $n \times n$ weight matrix $W$. We shall address the problem of evaluating the configurational weight of all particle rearrangements which end up with $\mathbf{k}_{n}$ particles in the different boxes, regardless of their type. Upon suitable normalization, for each Boltzmann weight matrix $W$, we shall use this to define the Gibbs canonical probability generating function of the random box-occupancies $\mathbf{K}_{n, k}=\left(K_{n, k}(m) ; m=1, . ., n\right)$, given a total population of $k$ particles. From this model, the event $\mathbf{K}_{n, k}=\mathbf{k}_{n}$ will be realized either because there are $k_{m}$ type- $m$ particles in box number $m$ but also for any rearrangement of this peculiar configuration, the weight of each being needed to evaluate its occurrence probability. After suitably randomizing the particle number $k$, we shall rather work with the grand-canonical probability generating function of the random occupancies $\mathbf{K}_{n, z}$ where 'fugacity' parameter $z$ is in one to one correspondence with the average number $\kappa$ of particles in the system. We shall show that it has a determinantal form and that the associated probabilities are rather permanantal, that is, can be expressed in terms of the permanent of some enlarged matrix derived from $W$. Since $W$ has non-negative entries, it turns out that the joint distribution of $\mathbf{K}_{n, z}$ is infinitely divisible (that is in the compound Poisson class). As a result, it makes sense to raise the probability generating function of $\mathbf{K}_{n, z}$ to the power $\alpha$, for all $\alpha \in(0, \infty)$. This leads to a fractional occupancy statistics of order $\alpha$, the special case $\alpha=1$ corresponding to the standard Bose-Einstein occupancy model. Under the additional condition that $W$ is definite non-negative, it makes sense to raise the probability generating function of $\mathbf{K}_{n, z}$ to the power $\alpha$, for all $\alpha \in\{. .,-2,-1\}$ : for such distributions, the maximal number of particles within each box cannot exceed $-\alpha$. The special case $\alpha=-1$ corresponds to the usual Fermi-Dirac occupancy model involving an exclusion principle: no more than one particle within each box. Therefore, for doubly non-negative weight matrices (that is, both non-negative and definite non-negative), fractional occupancies of all order $\alpha \in\{. .,-2,-1\} \cup(0, \infty)$ can be defined. In the limit $|\alpha| \nearrow \infty$, a Maxwell-Boltzmann occupancy model is found: grand-canonical box-occupancies turn out to be independent and Poisson distributed. All these allegations can easily be derived from the version of the MacMahon master theorem which is relevant to our balls-in-boxes context, respecting transition weights. We now briefly describe the content of the manuscript in more details. 
In Section 2, we start illustrating these ideas in the case where $W$ is a weight matrix with $\{0,1\}$-entries, starting with the flat matrix. Here the configurational weight of particle rearrangements simply counts the number of admissible rearrangements when transition from box $m$ to box $m^{\prime}$ is either forbidden or allowed, depending on whether $W_{m, m^{\prime}}$ is 0 or 1 . We discuss some examples in Sub-sections 2.1 and 2.2; some are solvable, some are more involved. Subsection 2.3 considers the full case of a real-valued weight matrix that is doubly non-negative. Here we compute for instance the factorial moments of $\mathbf{K}_{n, z}$, the 2 -boxes joint law of the occupancies $\left(K_{n, z}\left(m_{1}\right) ; K_{n, z}\left(m_{2}\right)\right)$, the marginal law of $K_{n, z}(m)$. We prove that the limiting distribution of $\mathbf{K}_{n, z}$ when $\alpha \nearrow \infty$ is the Maxwell-Boltzmann distribution. We characterize the limit law of $\mathbf{K}_{n, z} / \kappa$ when the expected number $\kappa$ of particles goes to infinity, in terms of a specific multivariate gamma distribution.

Section 3 is devoted to what is needed of MacMahon master theorem which is relevant to our balls-in-boxes context.

Finally, we shall address the following problem: Assume particles can only be placed in boxes in the positions $-\infty<x_{1} \leq x_{2} \leq . . \leq x_{n}<\infty$ on the real line. The box number of a particle now is the index of its position on $\mathbb{R}$ and the model is spatially extended: a particle is attached to box number $m$ if it stands at $m$-th position $x_{m}$. The purpose of Section 4 is to construct natural doubly non-negative weight matrices $W$ which are indexed by $\mathbf{x}:=\left(x_{1}, . ., x_{n}\right) \in \mathbb{R}^{n}$, representing particle positions on the line. They are adapted to the spatially extended version of the occupancy problem. In such models, the $m \times m^{\prime}$ entry $W_{m, m^{\prime}}$ of $W$ is of the form $W\left(x_{m}, x_{m^{\prime}}\right)$; using this, occupancy statistics can be considered when $-\log W\left(x_{m}, x_{m^{\prime}}\right)$ is now the energy required to move a particle from position $x_{m}$ to position $x_{m^{\prime}}$. The construction of such matrices parallels the one of non-negative correlation functions whose spectral measure is positive. We shall also discuss the relevance of the recent notion of an infinitely divisible weight matrix, in the context of our investigations. Several examples are supplied.

\section{Multi-type weighted particle rearrangements}

As discussed in the Introduction, we start with the simplest rearrangement case before extending the construction to more general weight matrices.

\subsection{The simple rearrangement case}

Suppose we are given a total amount of $k \geq 1$ labeled particles (items) of $n$ different types (or colors), with $n \geq 2$. Let $k_{m}, m=1, . ., n$ be the number of type- $m$ particles in some initial configuration, with $\left|\mathbf{k}_{n}\right|:=k_{1}+. .+k_{n}=k$. Place $k_{m}$ particles in box number $m, m=1, . ., n$, respectively. When considering the problem of enumerating the number of ways to permute these $\mathbf{k}_{n}$ particles ending 
up with $\mathbf{k}_{n}$ particles in the different boxes (or urns), the following generating function proves necessary

$$
\frac{1}{1-z\left(u_{1}+. .+u_{n}\right)}=\sum_{\mathbf{k}_{n} \in \mathbb{N}^{n}} z^{\left|\mathbf{k}_{n}\right|} \frac{\left|\mathbf{k}_{n}\right| !}{\prod_{m=1}^{n} k_{m} !} \prod_{m=1}^{n} u_{m}^{k_{m}} .
$$

Here $\mathbf{u}:=\left(u_{1}, . ., u_{n}\right) \in[0,1]^{n}$ 'marks' the different types of particles and $z \in\left[0, z_{c}:=1 / n\right)$ is a 'marker' of the total number of particles. From this, interpreting the above generating function as an 'exponential' generating function, extracting the Taylor coefficients in the variables $\prod_{m=1}^{n} u_{m}^{k_{m}}$ of its series expansion, as conventional wisdom suggests, there are $\left|\mathbf{k}_{n}\right| !=k$ ! ways to permute the $\mathbf{k}_{n}$ labeled (distinguishable) particles. In other words, if $\mathcal{S}\left(\mathbf{k}_{n}\right)$ is the set of all such permutations, then $\left|\mathcal{S}\left(\mathbf{k}_{n}\right)\right|=\left|\mathbf{k}_{n}\right|$ !. This is poorly informative, so far.

Would the particles be unlabeled within each type class, there would clearly be $\frac{\left|\mathbf{k}_{n}\right| !}{\prod_{m=1}^{n} k_{m} !}$ ways to permute the $\mathbf{k}_{n}$ unlabeled particles, looking the above generating function as an 'ordinary' generating function and simply extracting the coefficients in the variables $\prod_{m=1}^{n} u_{m}^{k_{m}}$ of its series expansion. In this case, a permutation is called a rearrangement of the word $1^{k_{1}} \ldots n^{k_{n}}$. Clearly, when assigning $u_{1}=. .=u_{n}=1$, the $\left[z^{k}\right]$-coefficient of $(1-z n)^{-1}$, which is $n^{k}$, will count the number of ways to permute (rearrange) $k$ unlabeled particles of $n$ different types, regardless of the number of particles within each class.

Assuming for example $n=2, k_{1}=2, k_{2}=1, k=3$, identifying the 2 particles of the first type, there are 3 different possible rearranged words out of $1^{2} 2^{1}$, namely 112,121 and 211 whereas there are $2^{3}=8$ ways to permute 3 unlabeled particles of 2 different types: $\emptyset 222$, (1 22, 2 12, 221$),(112,121,21$ $1)$ and $111 \emptyset$ corresponding respectively to the partitions $\left(k_{1}, k_{2}\right)=(0,3),(1,2)$, $(2,1)$ and $(3,0)$ of $k=3$. Here $\emptyset$ is the empty box 'word'. For more details on rearrangements, see Cartier and Foata, [5].

Let $W=J$ where $J$ is the $n \times n$ flat 'weight' matrix whose entries are all equal to 1 , for which $\operatorname{Spect}(W)=\{0, . .(n-1)$ times.., $0 ; n\}$. With $|W|:=\operatorname{det}(W)$ and $U:=\operatorname{diag}(\mathbf{u})$, it turns out that

$$
\frac{1}{1-z\left(u_{1}+. .+u_{n}\right)}=|I-z U W|^{-1} .
$$

Next, we note that

$$
\left[z^{\left|\mathbf{k}_{n}\right|} \prod_{m=1}^{n} \frac{u_{m}^{k_{m}}}{k_{m} !}\right] \log |I-z U W|^{-1}=\left(\left|\mathbf{k}_{n}\right|-1\right) !
$$

counts the number of ways to permute cyclically the $\mathbf{k}_{n}$ labeled particles, when restricting $\mathcal{S}\left(\mathbf{k}_{n}\right)$ to $\mathcal{S}_{c}\left(\mathbf{k}_{n}\right)$, the set of all connected (one cycle) permutations, clearly with $\left|\mathcal{S}_{c}\left(\mathbf{k}_{n}\right)\right|=\left(\left|\mathbf{k}_{n}\right|-1\right)$ !. 
With $\alpha>0$ now 'marking' the number of cycles, this suggests to also consider the generating function

$$
|I-z U W|^{-\alpha}=e^{-\alpha \log |I-z U W|} .
$$

With $p \in \mathbb{N}$, the generating function $\left[\alpha^{p}\right]|I-z U W|^{-\alpha}$ is the one counting the permutations of $n$ types of particles, when restricting them to be a collection of $p$ disconnected cycles. In particular, $[\alpha]|I-z U W|^{-\alpha}=-\log |I-z U W|$ counts the permutations of $n$ types of particles, when restricting them to be simple cycles. We shall return to this point later.

\section{Canonical and grand-canonical randomization.}

Let $\mathbf{K}_{n, k}:=\left(K_{n, k}(m) ; m=1, . ., n\right)$ denote an integral-valued random vector which will stand for occupancies of the boxes $m=1, . ., n$ given a total population of $k$ particles.

Given $\left|\mathbf{k}_{n}\right|=k$, define naturally the (conditional) probability that $K_{n, k}(m)=$ $k_{m}, m=1, . ., n$ by the ratio of their configurations numbers

$$
\mathbb{P}\left(\mathbf{K}_{n, k}=\mathbf{k}_{n}\right)=\frac{\left(\begin{array}{c}
k \\
k_{1} . . k_{n}
\end{array}\right)}{\left[z^{k}\right](1-z n)^{-1}} \cdot \mathbf{1}\left(\left|\mathbf{k}_{n}\right|=k\right) .
$$

The event $\mathbf{K}_{n, k}=\mathbf{k}_{n}$ will be realized either because there are $k_{m}$ type- $m$ particles in box number $m$ but also for any rearrangement of this peculiar configuration. In other words,

$$
\mathbb{E}\left(\prod_{m=1}^{n} u_{m}^{K_{n, k}(m)}\right)=\frac{\left[z^{k}\right]\left(1-z\left(u_{1}+. .+u_{n}\right)\right)^{-1}}{\left[z^{k}\right](1-z n)^{-1}}
$$

is its canonical (conditional) probability generating function.

To avoid considering the simplex $\left|\mathbf{k}_{n}\right|=k$, suppose one randomizes the total number $k$ of particles as follows: let there be a random number $K_{n, z}$ of particles where

$$
\mathbb{P}\left(K_{n, z}=k\right)=\frac{z^{k}\left[z^{k}\right](1-z n)^{-1}}{(1-z n)^{-1}} .
$$

In other words, with $u \in[0,1]$

$$
\mathbb{E}\left(u^{K_{n, z}}\right)=\frac{|I-z W|}{|I-z u W|}=\frac{1-z n}{1-z u n}
$$

is the (geometric) generating function of $K_{n, z}$. Then, parameters $z$ and $0<$ $\kappa:=\mathbb{E}\left(K_{n, z}\right)$ are related through

$$
\kappa:=\kappa(z)=\frac{z n}{1-z n},
$$


and, since 'fugacity' $z \in\left[0, z_{c}=1 / n\right)$, these are in one-to-one correspondence. Given $\kappa:=\mathbb{E}\left(K_{n, z}\right)=\frac{z n}{1-z n}$, define next the probability that $K_{n, z}(m)=k_{m}$, $m=1, . ., n$ simply by

$$
\mathbb{P}\left(\mathbf{K}_{n, z}=\mathbf{k}_{n}\right)=\frac{z^{\left|\mathbf{k}_{n}\right|}\left(\begin{array}{c}
\left|\mathbf{k}_{n}\right| \\
k_{1} . . k_{n}
\end{array}\right)}{(1-z n)^{-1}}, \mathbf{k}_{n} \in \mathbb{N}^{n} .
$$

In other words, with $W=J$

$$
\mathbb{E}\left(\prod_{m=1}^{n} u_{m}^{K_{n, z}(m)}\right)=\frac{\left(1-z\left(u_{1}+. .+u_{n}\right)\right)^{-1}}{(1-z n)^{-1}}=\frac{|I-z W|}{|I-z U W|}
$$

stands for its (grand canonical) probability generating function, now conditioned on the expected number $\kappa(z)$ of particles in the system.

Asymptotics for $\kappa \nearrow \infty$.

One suspects that, for fixed $n$, the following convergence in distribution will hold

$$
\frac{\mathbf{K}_{n, z}}{\kappa} \stackrel{d}{\rightarrow} \kappa / \infty \mathbf{X}_{n}
$$

where $\mathbf{X}_{n}:=(X(1), . ., X(n))$ is an $n$-dimensional random vector supported by $\mathbb{R}_{+}^{n}$. Let us prove this and characterize the joint law of $\mathbf{X}_{n}$. Recalling $\kappa:=\kappa(z)=\frac{z n}{1-z n}$, let us assume $z=(1-\epsilon) / n$ where, $\epsilon$ being close to $0^{+}, \kappa$, which is of order $\epsilon^{-1}$, tends to $\infty$. Then, with $\mathbf{s}:=\left(s_{1}, . ., s_{n}\right)$ and $S:=\operatorname{diag}(\mathbf{s})$

$$
\begin{aligned}
& \mathbb{E}\left(\prod_{m=1}^{n} e^{-s_{m} K_{n, z}(m) / \kappa}\right) \sim \mathbb{E}\left(\prod_{m=1}^{n} e^{-\epsilon s_{m} K_{n, n}-1(1-\epsilon)}(m)\right) \\
= & \frac{\epsilon}{1-(1-\epsilon)\left(\sum_{1}^{n} e^{-\epsilon s_{m}}\right) / n} \sim \frac{\epsilon}{1-(1-\epsilon)\left(n-\epsilon \sum_{1}^{n} s_{m}\right) / n} \\
& \sim \frac{1}{1+\left(\sum_{1}^{n} s_{m}\right) / n}=\frac{1}{\left|I+z_{c} S W\right|}=\mathbb{E}\left(\prod_{m=1}^{n} e^{-s_{m} X(m)}\right) .
\end{aligned}
$$

This is the Laplace Stieltjes transform of a symmetric exponentially distributed random vector $\mathbf{X}_{n}$, with 1-dimensional marginals $\mathbb{E}\left(e^{-s_{m} X(m)}\right)=\frac{1}{1+s_{m} / n}$, $m=1, . ., n$, the ones of exponentially distributed random variables on $\mathbb{R}_{+}$with mean $n^{-1}$.

Fractional statistics.

Let $z_{\alpha}:=z / \alpha$. We shall also be interested in random variables with distributions $\mathbb{P}_{\alpha}$ defined by

$$
\mathbb{E}_{\alpha}\left(\prod_{m=1}^{n} u_{m}^{K_{n, z}(m)}\right)=\frac{\left(1-\frac{z}{\alpha}\left(u_{1}+. .+u_{n}\right)\right)^{-\alpha}}{\left(1-\frac{z}{\alpha} n\right)^{-\alpha}}=\left(\frac{\left|I-z_{\alpha} W\right|}{\left|I-z_{\alpha} U W\right|}\right)^{\alpha}
$$


where $\alpha \in\{. .,-2,-1\} \cup(0, \infty)$ and $z<\alpha / n$ if $\alpha>0$ or $z \geq 0$ if $\alpha \in\{. .,-2,-1\}$. Since $W=J$, with $(\alpha)_{k}:=\alpha(\alpha+1) . .(\alpha+k-1)$, this is

$$
\mathbb{P}_{\alpha}\left(\mathbf{K}_{n, z}=\mathbf{k}_{n}\right)=\left(1-z_{\alpha} n\right)^{\alpha} \cdot z_{\alpha}^{\left|\mathbf{k}_{n}\right|} \frac{(\alpha)_{\left|\mathbf{k}_{n}\right|}}{\prod_{m=1}^{n} k_{m} !}, \mathbf{k}_{n} \in \mathbb{N}^{n} .
$$

When $\alpha=-i<0$, the range of $\mathbf{K}_{n, z}$ is $\mathbf{k}_{n} \in\{0, . ., i\}^{n}$. For instance, for $\mathbf{k}_{n} \in\{0,1\}^{n}, \mathbb{P}_{-1}\left(\mathbf{K}_{n, z}=\mathbf{k}_{n}\right)$ is the (Fermi-) joint probability to find one (respectively, no) particle in box number $m_{q}$ if $k_{m_{q}}=1$ (respectively, if $k_{m_{q}}=0$ ). Thus, when $W=J, \mathbf{K}_{n, z}$ is well-defined under $\mathbb{P}_{\alpha}$ for all $\alpha \in\{. .,-2,-1\} \cup(0, \infty)$. In particular,

$$
\mathbb{E}_{\alpha}\left(u^{K_{n, z}}\right)=\left(\frac{1-z_{\alpha} n}{1-z_{\alpha} u n}\right)^{\alpha}
$$

showing that $K_{n, z}$ has negative binomial $\left(\alpha, z_{\alpha} n\right)$ distribution (when $\alpha>0$ ) or multinomial $\left(-\alpha,\left(-z_{\alpha} n\right) /\left(1-n z_{\alpha}\right)\right)$ distribution $(\alpha \in\{. .,-2,-1\})$, with mean $\kappa:=\mathbb{E}_{\alpha}\left(K_{n, z}\right)=\frac{z n}{1-n z_{\alpha}}$. Such $\alpha$-distributions may be thought of as fractional occupancy statistics of order $\alpha$. When $\alpha=-1, \alpha=+1,|\alpha| \nearrow \infty$ we get a usual Fermi-Dirac, Bose-Einstein or Maxwell-Boltzmann distribution for $\mathbf{K}_{n, z}$, respectively. When $\alpha=k / 2$ where $k$ is any integer, we get a para-Boson statistics (see Aringazin et al and Greenberg et al, [1] and [14]). One of the questions raised next is: for which $W$ is $\mathbf{K}_{n, z}$ well-defined for all $\alpha$ in the positivity spectrum $\{. .,-2,-1\} \cup(0, \infty)$ ?

\subsection{Weighted restricted rearrangements: $\{0,1\}$-weight ma- trix}

These elementary considerations suggest first to introduce more generally the quantities $|I-z U W|^{-1}$ where $W$ is now a $n \times n$ weight matrix whose entries all belong to $\{0,1\}$. By doing so, we address the problem of enumerating the number of ways to permute the $\left|\mathbf{k}_{n}\right|=k$ particles when the transition from type $m$ to type $m^{\prime}$ is allowed at the only condition that entry $W_{m, m^{\prime}}=1$. Define formally energy (cost) of transition $m \rightarrow m^{\prime}$ as $H_{m, m^{\prime}}:=-\log W_{m, m^{\prime}}$. Then, would $W_{m, m^{\prime}}=0$, would the transition from type $m$ to type $m^{\prime}$ be forbidden as the energy required to realize this transition is infinite. Would $W_{m, m^{\prime}}=1$, the transition $m \rightarrow m^{\prime}$ requires no particular energy. Proceeding in this way, the random occupancies $\mathbf{K}_{n, z}$ are now dictated by the weighted restricted rearrangements encoded by $W$. For related problems, see Diaconis et al, [9].

The quantity $|I-z U W|^{-1}$ is defined for $\mathbf{u}:=\left(u_{1}, . ., u_{n}\right) \in[0,1]^{n}$ and $z \in$ $\left[0, z_{c}:=1 / \rho(W)\right)$ where, with $\lambda_{m} ; m=1, . ., n$ the spectrum of $W, \rho(W)=$ $\|W\|=\max \left(\left|\lambda_{m}\right| ; m=1, . ., n\right)$ is the spectral radius of $W$. The critical fugacity 
$z_{c}$ is the reciprocal of the spectral radius of $W$ and $|I-z U W|^{-1}$ first becomes singular at $z_{c}$. Since all such $W$ are non-negative, by Perron-Foebenius theorem, the eigenvalue with largest modulus is real. Since $W$ only has $\{0,1\}$-entries, $\rho(W) \in[1, n]$ and so $z_{c} \in[1 / n, 1]$. The weighted random occupancies $K_{n, z}(m)$, $m=1, . ., n$ can be defined accordingly. As we shall indeed see later in the next section, such random variables are well-defined; in fact they are always well-defined as soon as $W$ has non-negative entries. This is because the Taylor coefficients in the variables $\prod_{m=1}^{n} u_{m}^{k_{m}}$ of the series expansion of $\frac{|I-z W|}{|I-z U W|}$ are the permanents $\operatorname{Per}\left(W\left(\mathbf{k}_{n}\right)\right)$. Here $W\left(\mathbf{k}_{n}\right)$ are the $\left|\mathbf{k}_{n}\right| \times\left|\mathbf{k}_{n}\right|$ matrix obtained by repeating (removing and deleting) each $W_{m, m^{\prime}}$ into a size $k_{m} \times k_{m^{\prime}}$ block if $k_{m}$ and $k_{m^{\prime}}$ both positive (otherwise). Permanents of a matrix with non-negative entries are non-negative. Note that the basic quantity appearing in the law of $K_{n, z}$ reads $|I-z W|^{-1}=\prod_{m=1}^{n}\left(1-z \lambda_{m}\right)^{-1}$.

Similarly, random variables $\mathbf{K}_{n, z}$ with law $\mathbb{P}_{\alpha}$ parameterized by $\alpha>0$ are well-defined for all $\alpha>0$ when $W$ has in particular non-negative entries. This is because the Taylor coefficients in the variables $\prod_{m=1}^{n} u_{m}^{k_{m}}$ of the series expansion of $\left(\frac{\left|I-z_{\alpha} W\right|}{\left|I-z_{\alpha} U W\right|}\right)^{\alpha}$ now are proportional to the $\alpha$-permanents $\operatorname{Per}_{\alpha}\left(W\left(\mathbf{k}_{n}\right)\right)$. $\alpha$-Permanents of a matrix with non-negative entries are non-negative (see the next Section). In all such cases, random variables $\mathbf{K}_{n, z}$ with law $\mathbb{P}_{\alpha}$ (where $\alpha>0)$ are infinitely divisible, that is in the compound Poisson class. When $\alpha \in\{. .,-2,-1\}$, a necessary and sufficient condition for $\mathbf{K}_{n, z}$ with law $\mathbb{P}_{\alpha}$ to be well-defined is that $W$ has all its $2^{n}$ principal minors non-negative (see VereJones, [21], Prop. 6.1 for the necessity condition). If $\mathbf{K}_{n, z}$ with law $\mathbb{P}_{\alpha}$ is to be defined for all $\alpha \in\{. .,-2,-1\} \cup(0, \infty)$, a sufficient condition is that $W$ it has all its principal minors non-negative and is non-negative. If $W$ is in addition symmetric, then a doubly non-negative $W$ would do (doubly non-negative matrices are those which are both definite non-negative and with non-negative entries).

\section{Examples.}

- Assume $W=I$. Then, with $z<z_{c}=1$

$$
|I-z U W|^{-1}=\prod_{m=1}^{n}\left(1-z u_{m}\right)^{-1}=\frac{1}{1-\oplus_{m=1}^{n}\left(z u_{m}\right)}
$$

where $x_{1} \oplus x_{2}=x_{1}+x_{2}-x_{1} x_{2}$ is the commutative and associative probabilistic sum. Here,

$$
\left[z^{\left|\mathbf{k}_{n}\right|} \prod_{m=1}^{n} \frac{u_{m}^{k_{m}}}{k_{m} !}\right]|I-z U W|^{-1}=\prod_{m=1}^{n} k_{m} !
$$

counts the number of ways to permute the $\mathbf{k}_{n}$ labeled particles when the image 
particle is forced to return back to its own type. Therefore,

$$
\mathbb{E}\left(u^{K_{n, z}}\right)=\frac{|I-z W|}{|I-z u W|}=\left(\frac{1-z}{1-z u}\right)^{n}
$$

is the generating function of $K_{n, z}$, with mean $\kappa=(n z) /(1-z)$. The $z^{k}$-coefficient of $(1-z)^{-n}$ is $\left(\begin{array}{c}n+k-1 \\ k\end{array}\right)$.

Clearly, in this interaction-free case

$$
\frac{\mathbf{K}_{n, z}}{\kappa} \stackrel{d}{\rightarrow} / \infty \mathbf{X}_{n}
$$

where the components of the random vector $\mathbf{X}_{n}$ are independent and identically distributed (iid), mean 1 , exponentially distributed random variables on $\mathbb{R}_{+}$.

- In the last two examples, the weight matrix is definite non-negative. It has all principal minors non-negative. The canonical form of a $\{0,1\}$-valued definite non-negative weight matrix $W$ is made of $p$ flat Jordan blocks $J_{q}$ (of type $J), q=1, . ., p$ where the sizes of $J_{q}$ are $n_{q} \times n_{q}, q=1, . . p$ with $\sum_{q=1}^{p} n_{q}=n$ and $1 \leq n_{1} \leq \ldots \leq n_{p}$. Since $W$ is symmetric, outside the Jordan blocks, $W$ has zero entries and so is block-diagonal. The number of such matrices therefore is the number of partitions of $n$. The spectrum of $W$ is $\operatorname{Spect}(W)=$ $\cup_{q=1}^{p}\left\{0, . .\left(n_{q}-1\right)\right.$ times.., $\left.0 ; n_{q}\right\}$ and with $n_{0}:=0$ and $\bar{n}_{q}:=\sum_{r=0}^{q} n_{q}, q=$ $0, . ., p$

$$
\begin{gathered}
|I-z U W|^{-1}=\prod_{q=1}^{p}\left(1-z\left(u_{\bar{n}_{q-1}+1}+. .+u_{\bar{n}_{q}}\right)\right)^{-1} \\
=\left[1-\oplus_{q=1}^{p}\left(z\left(u_{\bar{n}_{q-1}+1}+. .+u_{\bar{n}_{q}}\right)\right)\right]^{-1}
\end{gathered}
$$

defined for $z<1 / n_{p}$. Representing the above partition sequence $1 \leq n_{1} \leq \ldots \leq$ $n_{p}$ by the sequence $0=: m_{0}<1 \leq m_{1}<\ldots<m_{r}$ where each $m_{q}, q=1, . ., r$ has multiplicity $d_{q}$ now with $\sum_{q=1}^{r} d_{q} m_{q}=n$,

$$
\mathbb{E}\left(u^{K_{n, z}}\right)=\frac{|I-z W|}{|I-z u W|}=\prod_{q=1}^{r}\left(\frac{1-z m_{q}}{1-z u m_{q}}\right)^{d_{q}}
$$

is the generating function of $K_{n, z}$, with mean

$$
\kappa=\sum_{q=1}^{r}\left(z d_{q} m_{q}\right) /\left(1-z m_{q}\right) .
$$

The first introductory example $(W=J)$ corresponds to an irreducible case with $r=1, m_{1}=n$ and $d_{1}=1$ whereas the second example $(W=I)$ is completely reducible with $r=1, m_{1}=1$ and $d_{1}=n$.

For this class of $W, \mathbf{K}_{n, z}$ is well-defined under $\mathbb{P}_{\alpha}$ for all $\alpha \in\{. .,-2,-1\} \cup$ $(0, \infty)$. 
- (Derangements) Assume now that $W=J-I$ which is symmetric but not definite-positive.

One can check that $\operatorname{Spect}(W)=\{-1, . .(n-1)$ times.., $-1 ; n-1\}$. Then, with $z<z_{c}=1 /(n-1)$, it holds that

$$
|I-z U W|^{-1}=\frac{1}{1-\sum_{p=2}^{n} z^{p}(p-1) \sigma_{p}(\mathbf{u})}
$$

where

$$
\sigma_{p}(\mathbf{u})=\sum_{1 \leq m_{1}<. .<m_{p} \leq n} \prod_{q=1}^{p} u_{m_{q}}
$$

are the elementary symmetric functions. For this example,

$$
\left[z^{\left|\mathbf{k}_{n}\right|} \prod_{m=1}^{n} \frac{u_{m}^{k_{m}}}{k_{m} !}\right]|I-z U W|^{-1}
$$

counts the number of ways to permute the $\mathbf{k}_{n}$ labeled particles, when no permuted particle is allowed to return to its own type. A permutation in this class is called a derangement (see Gillis, [13]).

In this case, with $0 \leq z<1 / \rho(W)=1 /(n-1)$, we get

$$
|I-z W|^{-1}=\frac{1}{1-\sum_{p=2}^{n} z^{p}(p-1)\left(\begin{array}{l}
n \\
p
\end{array}\right)}=\frac{1}{(1+z)^{n-1}[1-(n-1) z]}
$$

with $\left[z^{k}\right]|I-z W|^{-1}=\sum_{l=0}^{k}(-1)^{l}\left(\begin{array}{c}n+l-2 \\ l\end{array}\right)(n-1)^{k-l} \geq 0$. So, for instance

$$
\mathbb{E}\left(u^{K_{n, z}}\right)=\frac{|I-z W|}{|I-z u W|}=\left(\frac{1+z}{1+z u}\right)^{n-1} \cdot \frac{1-(n-1) z}{1-(n-1) z u}
$$

is the generating function of $K_{n, z}$, with mean

$$
\kappa=(n-1) z\left[\frac{1}{1-(n-1) z}-\frac{z}{1+z}\right] .
$$

For this class of $W, \mathbf{K}_{n, z}$ is well-defined under $\mathbb{P}_{\alpha}$ for all $\alpha \in(0, \infty)$ (see Foata and Zeilberger, [12]) but, since the principal minors can be negative, not for $\alpha \in\{. .,-2,-1\}$.

- If $W$ is any of the $(n-1)$ ! orthogonal matrices of a cyclic (connected) permutation

$$
|I-z U W|^{-1}=\frac{1}{1-\prod_{m=1}^{n}\left(z u_{m}\right)}, z<z_{c}=1 .
$$

For this example, there is no way to permute the $\mathbf{k}_{n}$ labeled particles, unless all $k_{m}=k_{1}, m=1, . ., n$ in which case this number is $\left(k_{1} !\right)^{n}$. Here,

$$
\mathbb{E}\left(u^{K_{n, z}}\right)=\frac{|I-z W|}{|I-z u W|}=\frac{1-z^{n}}{1-(z u)^{n}}
$$


is the generating function of $K_{n, z}$, with mean $\kappa=\left(n z^{n}\right) /\left(1-z^{n}\right)$. Note that the spectrum of $W$ consists in the $n$ roots of unity. With $\lambda_{m}=e^{2 i \pi(m-1) / n}$, $m=1, . ., n$ note the identity $\sum_{m=1}^{n} \frac{z \lambda_{m}}{1-z \lambda_{m}}=\frac{n z^{n}}{1-z^{n}}=\kappa$.

Clearly, in this case

$$
\frac{\mathbf{K}_{n, z}}{\kappa} \stackrel{d}{\rightarrow} \kappa / \infty \mathbf{X}_{n}
$$

where, with $\mathbb{E}\left(\prod_{m=1}^{n} e^{-s_{m} X(m)}\right)=\left(1+\left(\sum_{1}^{n} s_{m}\right) / n\right)^{-1}, \mathbf{X}_{n}$ has symmetric multivariate exponential distribution.

For this class of $W, \mathbf{K}_{n, z}$ is well-defined under $\mathbb{P}_{\alpha}$ for all $\alpha \in(0, \infty)$ but, since the principal minors can be negative, not for $\alpha \in\{. .,-2,-1\}$.

The next two examples are more involved and would require some additional knowledge of the weight matrix spectrum:

- (periodic nearest neighbors interactions):

Assume $W_{1,2}=W_{1, n}=1 ; W_{m, m+1}=W_{m, m-1}=1, m=2, . ., n-1$ and $W_{n, 1}=W_{n, n-1}=1$, the other entries being all equal to 0 . In this case, $\rho(W)=2$ and $z_{c}=1 / 2$. Matrix $W$ is symmetric with real eigenvalues. In a standard (non-periodic) nearest neighbors interactions model, $W_{1, n}=W_{n, 1}=0$ and $W$ is strictly tridiagonal.

- tournaments: assume $W_{m, m}=0, m=1, . ., n$. For all $m^{\prime}>m$, fix $W_{m, m^{\prime}} \in\{0,1\}$ and force $W_{m^{\prime}, m}=1-W_{m, m^{\prime}}$ so that $\sum_{m, m^{\prime}} W_{m, m^{\prime}}=\left(\begin{array}{c}n \\ 2\end{array}\right)$. Each $W$ fulfills $W+W^{\prime}=J-I$. There are $2^{n(n-1) / 2}$ tournaments as issues of pair matching games with $n$ players.

\section{Remark}

The distribution of $\mathbf{K}_{n, z}$ is exchangeable if and only if $\mathbb{E}\left(\prod_{m=1}^{n} u_{m}^{K_{n, z}(m)}\right)=$ $\mathbb{E}\left(\prod_{m=1}^{n} u_{\sigma_{m}}^{K_{n, z}(m)}\right)$ for all permutation $\sigma$ of $\{1, . ., n\}$; in other words, if and only if $|I-z U W|$ is a symmetric function of $\mathbf{u}:=\left(u_{1}, . ., u_{n}\right)$. This will be the case under the following special conditions: $W=a I+b(J-I)$ where $a$ and $b$ belong to $\{0,1\}$. Thus, when $W=I, W=J-I$ or $W=J$. And also when $W$ is the weight matrix of a cyclic (connected) permutation. In all these cases, for each order, all principal minors of $W$ coincide, which is a necessary and sufficient condition for the distribution of $\mathbf{K}_{n, z}$ to be exchangeable. This is clear from the development of $|I-z U W|$ in terms of all principal minors of $W$.

\subsection{Rearrangements from doubly non-negative weight ma- trices}

Let $W$ be an arbitrary weight matrix with real non-negative entries and with non-negative principal minors. With $z_{\alpha}:=z / \alpha$, we are interested in random 
variables $\mathbf{K}_{n, z}$ with probability generating functions

$$
\mathbb{E}_{\alpha}\left(\prod_{m=1}^{n} u_{m}^{K_{n, z}(m)}\right)=\left(\frac{\left|I-z_{\alpha} W\right|}{\left|I-z_{\alpha} U W\right|}\right)^{\alpha}
$$

where $\alpha \in\{. .,-2,-1\} \cup(0, \infty)$ and $z<\alpha / n$ if $\alpha>0$ or $z \geq 0$ if $\alpha \in\{. .,-2,-1\}$. These random variables are well-defined for all $\alpha$ in the prescribed range and infinitely divisible when $\alpha>0$. They correspond to occupancy $\alpha$-distributions arising from rearrangements with weight $W$. If $W$ is in addition symmetric, then $W$ belongs to the class of doubly non-negative matrices.

For general doubly non-negative real-valued matrices, $\mathbf{K}_{n, z}$ is well-defined under $\mathbb{P}_{\alpha}$ where $\alpha$ varies in the announced range.

Factorial moments of $\mathbf{K}_{n, z}$.

Let $k_{m} \geq 0, m=1, . ., n$ and $\{n\}_{k}:=n(n-1) . .(n-k+1),\{n\}_{0}:=1$. Define the factorial moments of $\mathbf{K}_{n, z}$ to be

$$
\mu_{\alpha}\left(\mathbf{k}_{n}\right):=\mathbb{E}_{\alpha}\left(\prod_{m=1}^{n}\left\{K_{n, z}(m)\right\}_{k_{m}}\right) .
$$

We have

$$
\begin{gathered}
\mathbb{E}_{\alpha}\left(\prod_{m=1}^{n} u_{m}^{K_{n, z}(m)}\right)=\left(\frac{\left|I-z_{\alpha} W\right|}{\left|I-z_{\alpha} U W\right|}\right)^{\alpha}=\left(\frac{\left|I-z_{\alpha} W\right|}{\left|I-z_{\alpha} W-z_{\alpha}(U-I) W\right|}\right)^{\alpha} \\
=\left(\left|I-(U-I) z_{\alpha} W\left(I-z_{\alpha} W\right)^{-1}\right|\right)^{-\alpha}=:\left(\left|I-(U-I) W_{z_{\alpha}}\right|\right)^{-\alpha} .
\end{gathered}
$$

Therefore, the factorial moments $\mu_{\alpha}\left(\mathbf{k}_{n}\right)$ of $\mathbf{K}_{n, z}$ exist; they are given by the Taylor coefficients in the variables $\prod_{m=1}^{n}\left(u_{m}-1\right)^{k_{m}}$ of the series expansion of $\left(\left|I-(U-I) W_{z_{\alpha}}\right|\right)^{-\alpha}$, namely $\operatorname{Per}_{\alpha}\left(W_{z_{\alpha}}\left(\mathbf{k}_{n}\right)\right)$, where $W_{z_{\alpha}}=z_{\alpha} W\left(I-z_{\alpha} W\right)^{-1}$ is the resolvent matrix of $W$. If $W$ has non-negative entries, so does $W_{z_{\alpha}}$ and therefore the factorial moments of $\mathbf{K}_{n, z}$, namely $\operatorname{Per}_{\alpha}\left(W_{z_{\alpha}}\left(\mathbf{k}_{n}\right)\right)$ are nonnegative. If $W$ is definite-positive with positive eigenvalues $\lambda_{m}$, so does $W_{z_{\alpha}}$ with eigenvalues $\left(z_{\alpha} \lambda_{m}\right) /\left(1-z_{\alpha} \lambda_{m}\right)$. For doubly non-negative weight matrices, $\mu_{\alpha}\left(\mathbf{k}_{n}\right)$ are well defined for all $\alpha$ in the full range $\{. .,-2,-1\} \cup(0, \infty)$.

Marginal distribution of $K_{n, z}(m)$.

With $U_{m}$ a diagonal matrix whose $m \times m$ entry is $u_{m}$, all other diagonal entries being 1 , with $z_{\alpha}:=z / \alpha$, we have

$$
\begin{gathered}
\mathbb{E}_{\alpha}\left(u_{m}^{K_{n, z}(m)}\right)=\left(\frac{\left|I-z_{\alpha} W\right|}{\left|I-z_{\alpha} U_{m} W\right|}\right)^{\alpha}=\left(\frac{\left|I-z_{\alpha} W\right|}{\left|I-z_{\alpha} W-z_{\alpha}\left(U_{m}-I\right) W\right|}\right)^{\alpha} \\
=\left(\left|I-\left(U_{m}-I\right) z_{\alpha} W\left(I-z_{\alpha} W\right)^{-1}\right|\right)^{-\alpha}=\left(\left|I-\left(U_{m}-I\right) W_{z_{\alpha}}\right|\right)^{-\alpha}
\end{gathered}
$$




$$
=\left(1-\left(u_{m}-1\right)\left(W_{z_{\alpha}}\right)_{m, m}\right)^{-\alpha}=:\left(\frac{1-p_{m}}{1-p_{m} u_{m}}\right)^{\alpha} .
$$

Since $\left(W_{z_{\alpha}}\right)_{m, m} \geq 0$, when $\alpha>0$, this is the distribution of a negative binomial random variable with parameters $\left(\alpha, p_{m}:=\frac{\left(W_{z_{\alpha}}\right)_{m, m}}{1+\left(W_{z_{\alpha}}\right)_{m, m}}\right)$. Here $p_{m}$ is the success probability depending on $z$ and $\alpha$. When $\alpha \in\{. .,-2,-1\}$, this generating function is the one of a multinomial distribution with parameters $\left(-\alpha, \pi_{m}:=-\left(W_{z_{\alpha}}\right)_{m, m}\right)$ where $\pi_{m}$ now is the probability that the underlying Bernoulli random variable takes the value 1 .

Note from this analysis that in any case for $\alpha$

$$
\kappa:=\mathbb{E}_{\alpha}\left(K_{n, z}\right)=\alpha \sum_{m=1}^{n}\left(W_{z_{\alpha}}\right)_{m, m}=\alpha \cdot \operatorname{tr}\left(W_{z_{\alpha}}\right) .
$$

Joint distribution of $\left(K_{n, z}\left(m_{1}\right), K_{n, z}\left(m_{2}\right)\right)$.

Let $1 \leq m_{1}<m_{2} \leq n$. With $U_{m_{1}, m_{2}}$ a diagonal matrix whose entries $m_{1} \times m_{1}$ and $m_{2} \times m_{2}$ are $u_{m_{1}}$ and $u_{m_{2}}$, all other diagonal entries being 1 , we have

$\mathbb{E}_{\alpha}\left(u_{m_{1}}^{K_{n, z}\left(m_{1}\right)} u_{m_{2}}^{K_{n, z}\left(m_{2}\right)}\right)=\left(\frac{\left|I-z_{\alpha} W\right|}{\left|I-z_{\alpha} U_{m_{1}, m_{2}} W\right|}\right)^{\alpha}=\left(\left|I-\left(U_{m_{1}, m_{2}}-I\right) W_{z_{\alpha}}\right|\right)^{-\alpha}$

$=\left(1-\sum_{i \in\{1,2\}}\left(u_{m_{i}}-1\right)\left(W_{z_{\alpha}}\right)_{m_{i}, m_{i}}+\left(u_{m_{1}}-1\right)\left(u_{m_{2}}-1\right)\left|W_{z_{\alpha}}\left(m_{1}, m_{2}\right)\right|\right)^{-\alpha}$

where $\left|W_{z_{\alpha}}\left(m_{1}, m_{2}\right)\right|$ is the corresponding minor of $W_{z_{\alpha}}$. From this, we get in particular

$$
\begin{gathered}
\operatorname{Cov}_{\alpha}\left(K_{n, z}\left(m_{1}\right), K_{n, z}\left(m_{2}\right)\right)=\alpha\left[\left(W_{z_{\alpha}}\right)_{m_{1}, m_{1}}\left(W_{z_{\alpha}}\right)_{m_{2}, m_{2}}-\left|W_{z_{\alpha}}\left(m_{1}, m_{2}\right)\right|\right] \\
=\alpha\left(W_{z_{\alpha}}\right)_{m_{1}, m_{2}}\left(W_{z_{\alpha}}\right)_{m_{2}, m_{1}}
\end{gathered}
$$

which is non-negative when $W_{z_{\alpha}}$ (or $W$ ) is non-negative or when $W_{z_{\alpha}}$ (or $W$ ) is symmetric (in particular definite positive). When $\alpha>0,\left(K_{n, z}\left(m_{1}\right), K_{n, z}\left(m_{2}\right)\right)$ are positively correlated. Note that the above covariance decreases with $\alpha$ and that $\operatorname{Cov}_{\alpha}\left(K_{n, z}\left(m_{1}\right), K_{n, z}\left(m_{2}\right)\right) \underset{\alpha{ }^{\prime} \infty}{0}$. In sharp contrast, when $\alpha \in$ $\{. .,-2,-1\}$, these random variables are negatively correlated.

Limiting distribution of $\mathbf{K}_{n, z}$ when $\alpha \nearrow \infty$.

To the first order in $\alpha$, the exponential-trace expression of the determinant gives

$$
\mathbb{E}_{\alpha}\left(\prod_{m=1}^{n} u_{m}^{K_{n, z}(m)}\right)=\left(\frac{\left|I-z_{\alpha} W\right|}{\left|I-z_{\alpha} U W\right|}\right)^{\alpha} \underset{\alpha}{\longrightarrow_{\infty}}
$$




$$
e^{-\operatorname{tr}(z W)+\operatorname{tr}(z U W)}=\prod_{m=1}^{n} e^{-z\left(1-u_{m}\right) W_{m, m}} .
$$

This shows that asymptotically

$$
\mathbf{K}_{n, z} \underset{\alpha \nearrow \infty}{\stackrel{d}{\longrightarrow}} \mathbf{P}_{n, z}
$$

where $\mathbf{P}_{n, z}:=\left(P_{n, z}(1), . ., P_{n, z}(n)\right)$ are mutually independent Poisson distributed random variables with respective intensities $z W_{m, m}$, proportional to the diagonal terms of $W$. This is the limiting Maxwell-Boltzmann distribution for $\mathbf{K}_{n, z}$.

\section{Limit law of $\mathbf{K}_{n, z} / \kappa$ when $\kappa \nearrow \infty$.}

Assume first $\alpha=1$ and let $z_{c}:=1 / \rho(W)$. Assume $W$ differs from the identity. Letting $z:=z_{c}(1-\epsilon), \kappa=\operatorname{tr}\left(W_{z}\right)$ grows like $\epsilon^{-1}$, to the first order in $\epsilon$. This is because $\kappa=\sum_{1}^{n}\left(z \lambda_{m}\right) /\left(1-z \lambda_{m}\right)$ where $\left(\lambda_{m} ; m=1, . ., n\right)$ are the eigenvalues of $W$ with $\rho(W)$ the largest (real) of these: this model does not show up phase transition phenomena because $\kappa:=\kappa(z)$ is always divergent at critical fugacity $z_{c}$.

Now, with $\mathbf{s}:=\left(s_{1}, . ., s_{n}\right)^{\prime}$ and $S:=\operatorname{diag}(\mathbf{s})$

$$
\begin{aligned}
& \mathbb{E}\left(\prod_{m=1}^{n} e^{-\epsilon s_{m} K_{n, z}(m)}\right) \underset{\epsilon \searrow 0}{\sim} \frac{\left|I-z_{c}(1-\epsilon) W\right|}{\left|I-z_{c}(1-\epsilon)(1-\epsilon S) W\right|} \\
& \underset{\epsilon \searrow 0}{\sim} \frac{\left|I-z_{c} W+\epsilon z_{c} W\right|}{\left|I-z_{c} W+\epsilon z_{c}(I+S) W\right|} .
\end{aligned}
$$

Observing $\left|I-z_{c} W\right|=0$, the exponential-trace expansion of the determinant gives in this singular case

$$
\left|I-z_{c} W+\epsilon z_{c} W\right| \underset{\epsilon \searrow 0}{\sim} \epsilon \cdot \operatorname{tr}\left(z_{c} W \cdot \operatorname{adj}\left(I-z_{c} W\right)\right)
$$

where $\operatorname{adj}(A)$ is the adjugate matrix of $A$. Thus

$\mathbb{E}\left(\prod_{m=1}^{n} e^{-\epsilon s_{m} K_{n, z}(m)}\right) \underset{\epsilon \searrow 0}{\longrightarrow} \frac{\operatorname{tr}\left(z_{c} W \cdot \operatorname{adj}\left(I-z_{c} W\right)\right)}{\operatorname{tr}\left((I+S) z_{c} W \cdot \operatorname{adj}\left(I-z_{c} W\right)\right)}=: \mathbb{E}\left(\prod_{m=1}^{n} e^{-s_{m} X_{n}(m)}\right)$.

This shows that asymptotically

$$
\frac{\mathbf{K}_{n, z}}{\kappa} \underset{\kappa \nearrow \infty}{\stackrel{d}{\longrightarrow}} \mathbf{X}_{n}
$$

where the law of $\mathbf{X}_{n}:=\left(X_{n}(1), . ., X_{n}(n)\right)$ is characterized by the above joint Laplace-Stieltjes transform. Developing the traces, with $\left\langle\mathbf{s}, \mathbf{X}_{n}\right\rangle$ the scalar product of $\mathbf{s} \geq \mathbf{0}$ and $\mathbf{X}_{n}$, we get more specifically

$$
\mathbb{E}\left(e^{-\left\langle\mathbf{s}, \mathbf{X}_{n}\right\rangle}\right)=\left(1+\sum_{m=1}^{n} s_{m} \mu_{m}\right)^{-1}=\left(1+\left\langle\mathbf{s}, \boldsymbol{\mu}_{n}\right\rangle\right)^{-1}
$$


where $\boldsymbol{\mu}_{n}:=\left(\mu_{1}, . ., \mu_{n}\right), \mu_{m}=b_{m} / \sum_{1}^{n} b_{k}$ and $b_{m}=\left(z_{c} W \cdot \operatorname{adj}\left(I-z_{c} W\right)\right)_{m, m}$, $m=1, . ., n$ are the diagonal terms of the matrix $z_{c} W \cdot \operatorname{adj}\left(I-z_{c} W\right)$. In any case, it holds that $\sum_{1}^{n} \mu_{m}=1$ so that $\sum_{1}^{n} X_{n}(m)$ has mean 1 exponential law. The marginals of $\mathbf{X}_{n}$ are exponentially distributed with mean $\mu_{m}$. Note that, unless some special conditions hold on $W$, the distributions of $\mathbf{K}_{n, z}$ and $\mathbf{X}_{n}$ are not exchangeable. The telling feature of $\mathbf{X}_{n}$-law is that any linear non-negative combination of its components remains exponentially distributed.

When $\alpha>0$, considering the model $\mathbf{K}_{n, z}$ now under $\mathbb{P}_{\alpha}$, similar arguments would show that, with $\kappa=\operatorname{tr}\left(W_{z_{\alpha}}\right)$

$$
\frac{\mathbf{K}_{n, z}}{\kappa} \underset{\kappa \nearrow \infty}{\stackrel{d}{\longrightarrow}} \mathbf{X}_{n}
$$

where the law of $\mathbf{X}_{n}$ is now a multivariate-gamma distribution given by

$$
\mathbb{E}\left(e^{-\left\langle\mathbf{s}, \mathbf{X}_{n}\right\rangle}\right)=\left(1+\sum_{m=1}^{n} s_{m} \mu_{m}\right)^{-\alpha}=\left(1+\left\langle\mathbf{s}, \boldsymbol{\mu}_{n}\right\rangle\right)^{-\alpha}
$$

where $\boldsymbol{\mu}_{n}$ is now given in terms of the following $b_{m}$ :

$$
b_{m}=\left(z_{c, \alpha} W \cdot \operatorname{adj}\left(I-z_{c, \alpha} W\right)\right)_{m, m}, m=1, . ., n
$$

with $z_{c, \alpha}:=z_{c} / \alpha$. The latter Laplace-Stieltjes transform of $\mathbf{X}_{n}$ is well-defined because for all $\mathbf{s}>\mathbf{0}$, all $\lambda>0, \mathbb{E}\left(e^{-\lambda\left\langle\mathbf{s}, \mathbf{X}_{n}\right\rangle}\right)$, which is the Laplace-Stieltjes transform of the positive scalar random variable $\left\langle\mathbf{s}, \mathbf{X}_{n}\right\rangle$, is a Bernstein (completely monotone) function, of gamma type. The general shape of the moment generating function of a multivariate gamma random variable $\mathbf{X}_{n}$ is determinantal such as: $\mathbb{E}\left(e^{-\left\langle\mathbf{s}, \mathbf{X}_{n}\right\rangle}\right)=|I+S M|^{-\alpha}$ for some admissible matrix $M$. In our case, $M$ takes the particular form: $M=\left[\mu_{1} \mathbf{1}, . ., \mu_{n} \mathbf{1}\right]$ where $\mathbf{1}:=(1, . ., 1)^{\prime}$. The spectrum of such matrices is $\left\{0, . .(n-1)\right.$ times.., $\left.0 ; \sum_{1}^{n} \mu_{m}=1\right\}$ with nonnegative real eigenvalues only; by Proposition 4.6 of Vere-Jones, [21], it gives a well-defined Laplace-Stieltjes transform.

\section{Combinatorics related to MacMahon Master Theorem}

In this Section, we shall develop some combinatorial aspects of the MacMahon master theorem which are useful to our purposes. We shall let $\mathbb{N}=\{0,1,2, .$.$\} ,$ $\mathbb{N}_{*}=\{1,2, .$.$\} .$

We shall assume that $W$ is an $n \times n$ non-negative weight matrix, with $|W|=$ $\operatorname{det}(W)$. We shall let PerW stand for the permanent of $W$. We recall that $\mathbf{k}_{n}=\left(k_{1}, . ., k_{n}\right) \in \mathbb{N}^{n},\left|\mathbf{k}_{n}\right|=k_{1}+. .+k_{n} . \mathbf{u}=\left(u_{1}, . ., u_{n}\right) \in[0,1]^{n}$ and that $U=\operatorname{diag}(\mathbf{u}), I=$ Identity. Define $W\left(\mathbf{k}_{n}\right)$ to be the $\left|\mathbf{k}_{n}\right| \times\left|\mathbf{k}_{n}\right|$ matrix obtained 
by repeating (removing and deleting) each $W_{m, m^{\prime}}$ into a size $k_{m} \times k_{m^{\prime}}$ block if $k_{m}$ and $k_{m^{\prime}}$ both positive (otherwise). Assuming that $z \in[0,1 / \rho(W)$ ), we have

$$
\begin{aligned}
|I-z U W|^{-1} & =\sum_{\mathbf{k}_{n} \in \mathbb{N}^{n}} z^{\left|\mathbf{k}_{n}\right|} \prod_{m=1}^{n} u_{m}^{k_{m}}\left[\prod_{m=1}^{n} u_{m}^{k_{m}}\right] \prod_{m=1}^{n}(W \mathbf{u})_{m}^{k_{m}} \\
& =\sum_{\mathbf{k}_{n} \in \mathbb{N}^{n}} z^{\left|\mathbf{k}_{n}\right|} \prod_{m=1}^{n} \frac{u_{m}^{k_{m}}}{k_{m} !} \operatorname{Per} W\left(\mathbf{k}_{n}\right) \\
= & \sum_{\mathbf{k}_{n} \in \mathbb{N}^{n}} z^{\left|\mathbf{k}_{n}\right|} \prod_{m=1}^{n} \frac{u_{m}^{k_{m}}}{k_{m} !} \sum_{\sigma \in \mathcal{S}\left(\mathbf{k}_{n}\right)} \prod_{m, l_{m}} W_{m, \sigma_{1}\left(m, l_{m}\right)}
\end{aligned}
$$

where, if $\sigma\left(m, l_{m}\right)=\left(m^{\prime}, l^{\prime}\right), l_{m}=1, . ., k_{m}, m=1, . ., n, \sigma_{1}\left(m, l_{m}\right)=m^{\prime}$ gives the type of the image of particle $l_{m}$ in the class $m$. In the formulae displayed above, the first identity is due to Mac-Mahon (see Comtet, [8], for a short proof). It took some time to realize that the Taylor coefficients in $\mathbf{u}$ of the above expression can in fact be identified to the permanent of $W\left(\mathbf{k}_{n}\right)$ which is the content of the subsequent expressions (see [21] and [22] for historical remarks and background).

Remark. If $W$ is diagonal, with positive diagonal entries $\left(\lambda_{m}, m=1, . ., n\right)$, one can check that $\operatorname{Per} W\left(\mathbf{k}_{n}\right)=\prod_{m=1}^{n}\left(k_{m} ! \lambda_{m}^{k_{m}}\right)$ so that, as required

$$
|I-z U W|^{-1}=\sum_{\mathbf{k}_{n} \in \mathbb{N}^{n}} z^{\left|\mathbf{k}_{n}\right|} \prod_{m=1}^{n} u_{m}^{k_{m}} \lambda_{m}^{k_{m}}=\prod_{m=1}^{n}\left(1-z u_{m} \lambda_{m}\right)^{-1} \diamond \diamond
$$

Let $\alpha>0$ or $\alpha \in\{-1,-2, \ldots\}$. Let $c y c(\sigma)$ be the number of cycles in $\sigma \in \mathcal{S}\left(\mathbf{k}_{n}\right)$. Then

$$
\begin{gathered}
|I-z U W|^{-\alpha}=\sum_{\mathbf{k}_{n} \in \mathbb{N}^{n}} z^{\left|\mathbf{k}_{n}\right|} \prod_{m=1}^{n} \frac{u_{m}^{k_{m}}}{k_{m} !} \operatorname{Per}_{\alpha} W\left(\mathbf{k}_{n}\right) \\
=\sum_{\mathbf{k}_{n} \in \mathbb{N}^{n}} z^{\left|\mathbf{k}_{n}\right|} \prod_{m=1}^{n} \frac{u_{m}^{k_{m}}}{k_{m} !} \sum_{\sigma \in \mathcal{S}\left(\mathbf{k}_{n}\right)} \alpha^{c y c(\sigma)} \prod_{m, l_{m}} W_{m, \sigma_{1}\left(m, l_{m}\right)} \\
=\sum_{\mathbf{k}_{n} \in \mathbb{N}^{n}}(\alpha z)^{\left|\mathbf{k}_{n}\right|} \prod_{m=1}^{n} \frac{u_{m}^{k_{m}}}{k_{m} !} \sum_{\sigma \in \mathcal{S}\left(\mathbf{k}_{n}\right)}\left(\frac{1}{\alpha}\right)^{\left|\mathbf{k}_{n}\right|-c y c(\sigma)} \prod_{m, l_{m}} W_{m, \sigma_{1}\left(m, l_{m}\right)} .
\end{gathered}
$$

Thus,

$$
\left|I-\frac{z}{\alpha} U W\right|^{-\alpha}=\sum_{\mathbf{k}_{n} \in \mathbb{N}^{n}} z^{\left|\mathbf{k}_{n}\right|} \prod_{m=1}^{n} \frac{u_{m}^{k_{m}}}{k_{m} !} \sum_{\sigma \in \mathcal{S}\left(\mathbf{k}_{n}\right)}\left(\frac{1}{\alpha}\right)^{\left|\mathbf{k}_{n}\right|-c y c(\sigma)} \prod_{m, l_{m}} W_{m, \sigma_{1}\left(m, l_{m}\right)}
$$


and

$$
\left|I-\frac{z}{\alpha} U W\right|^{-\alpha}=\frac{1}{1-\left(1-\left|I-\frac{z}{\alpha} U W\right|^{\alpha}\right)}
$$

with

$1-\left|I-\frac{z}{\alpha} U W\right|^{\alpha}=\sum_{\mathbf{k}_{n} \in \mathbb{N}^{n}}(-z)^{\left|\mathbf{k}_{n}\right|} \prod_{m=1}^{n} \frac{u_{m}^{k_{m}}}{k_{m} !} \sum_{\sigma \in \mathcal{S}\left(\mathbf{k}_{n}\right)}\left(\frac{-1}{\alpha}\right)^{\left|\mathbf{k}_{n}\right|-c y c(\sigma)} \prod_{m, l_{m}} W_{m, \sigma_{1}\left(m, l_{m}\right)}$.

Further, with 'tr' standing for trace, the weight function

$$
\sigma \rightarrow w_{\alpha}(\sigma)=\alpha^{c y c(\sigma)} \prod_{m, l_{m}} W_{m, \sigma_{1}\left(m, l_{m}\right)}
$$

being multiplicative (equal to the product of the weights over the connected components of $\sigma$ )

$$
\begin{gathered}
-\alpha \log \left|I-\frac{z}{\alpha} U W\right|=\sum_{k \geq 1}\left(\frac{1}{\alpha}\right)^{k-1} \frac{z^{k}}{k} \operatorname{tr}\left(\{U W\}^{k}\right)= \\
\sum_{\mathbf{k}_{n} \in \mathbb{N}_{*}^{n}} z^{\left|\mathbf{k}_{n}\right|} \prod_{m=1}^{n} \frac{u_{m}^{k_{m}}}{k_{m} !} \sum_{\sigma \in \mathcal{S}_{c}\left(\mathbf{k}_{n}\right)}\left(\frac{1}{\alpha}\right)^{\left|\mathbf{k}_{n}\right|-1} \prod_{m, l_{m}} W_{m, \sigma_{1}\left(m, l_{m}\right)}
\end{gathered}
$$

where $\mathcal{S}_{c}\left(\mathbf{k}_{n}\right)$ is the subset of connected permutations $\sigma$ from $\mathcal{S}\left(\mathbf{k}_{n}\right)$, satisfying $\operatorname{cyc}(\sigma)=1$ and $\sigma\left(n, k_{n}\right)=(1,1)$. Thus

$$
\left[z^{\left|\mathbf{k}_{n}\right|} \prod_{m=1}^{n} \frac{u_{m}^{k_{m}}}{k_{m} !}\right]\left\{-\alpha \log \left|I-\frac{z}{\alpha} U W\right|\right\}=\sum_{\sigma \in \mathcal{S}_{c}\left(\mathbf{k}_{n}\right)}\left(\frac{1}{\alpha}\right)^{\left|\mathbf{k}_{n}\right|-1} \prod_{m, l_{m}} W_{m, \sigma_{1}\left(m, l_{m}\right)}
$$

involves cyclic weight products $\prod_{m=1}^{n} \prod_{l_{m}=1}^{k_{m}} W_{m, \sigma_{1}\left(m, l_{m}\right)}$, since $\sigma \in \mathcal{S}_{c}\left(\mathbf{k}_{n}\right)$ with $\sigma_{1}\left(n, k_{n}\right)=1$. In particular,

$$
\left[z^{\left|\mathbf{k}_{n}\right|} \prod_{m=1}^{n} \frac{u_{m}^{k_{m}}}{k_{m} !}\right]\{-\log |I-z U W|\}=\sum_{\sigma \in \mathcal{S}_{c}\left(\mathbf{k}_{n}\right)} \prod_{m, l_{m}} W_{m, \sigma_{1}\left(m, l_{m}\right)}=w_{1}\left(\mathcal{S}_{c}\left(\mathbf{k}_{n}\right)\right) .
$$

If in particular $\alpha=-1$, then

$$
|I+z U W|=\sum_{\mathbf{k}_{n} \in \mathbb{N}^{n}} z^{\left|\mathbf{k}_{n}\right|} \prod_{m=1}^{n} \frac{u_{m}^{k_{m}}}{k_{m} !} \sum_{\sigma \in \mathcal{S}\left(\mathbf{k}_{n}\right)}(-1)^{\left|\mathbf{k}_{n}\right|-c y c(\sigma)} \prod_{m, l_{m}} W_{m, \sigma_{1}\left(m, l_{m}\right)}
$$

where

$$
\sum_{\sigma \in \mathcal{S}\left(\mathbf{k}_{n}\right)}(-1)^{\left|\mathbf{k}_{n}\right|-c y c(\sigma)} \prod_{m, l_{m}} W_{m, \sigma_{1}\left(m, l_{m}\right)}=\left|W\left(\mathbf{k}_{n}\right)\right|
$$


which is null unless $\mathbf{k}_{n} \in\{0,1\}^{n}$. Thus, with $\left|W\left(\left\{m_{1}, . ., m_{p}\right\}\right)\right|$ a principal minor of $W$

$$
|I+z U W|=1+\sum_{p=1}^{n} z^{p} \sum_{1 \leq m_{1}<. .<m_{p} \leq n} \prod_{q=1}^{p} u_{m_{q}}\left|W\left(\left\{m_{1}, . ., m_{p}\right\}\right)\right| .
$$

Note that

$$
1-|I-z U W|=\sum_{p=1}^{n}(-1)^{p-1} z^{p} \sum_{1 \leq m_{1}<. .<m_{p} \leq n} \prod_{q=1}^{p} u_{m_{q}}\left|W\left(\left\{m_{1}, . ., m_{p}\right\}\right)\right|
$$

so that

$$
|I-z U W|^{-1}=\frac{1}{1-(1-|I-z U W|)} .
$$

From these combinatorial developments, it is clear (from positivity of induced probabilities) that if $W$ is definite non-negative, the occupancies $\mathbf{K}_{n, z}$ are well-defined under $\mathbb{P}_{\alpha}$ for all $\alpha>0$ and infinitely divisible. If in addition $W$ is definite non-negative, all principal minors of $W$ are non-negative and so $\mathbf{K}_{n, z}$ are well-defined under $\mathbb{P}_{\alpha}$ for all $\alpha \in\{. .,-2,-1\}$. For more on permanents, determinants, MacMahon master theorem and the like, see [19], [2], [7], [21], [22] and [5].

\section{Doubly non-negative and infinitely divisible weight matrices. Spatially extended boxes.}

Recall a weight matrix which is both non-negative and non-negative definite is doubly non-negative matrix. As underlined before, doubly non-negative weight matrices play a special role in our occupancy problems.

A probabilitic construction of doubly non-negative weight matrices.

Let us first give a systematic construction which generates doubly nonnegative matrices. In the process, we shall obtain the spatially extended system on the real line discussed in the introduction.

Let $\left(X_{1}, X_{2}\right)$ be a pair of iid random variables on $\mathbb{R}$ with a density, say $\phi(x)$. Consider the random variable $X=X_{1}-X_{2}$. Its density, say $f(x)$, exists and is given by $f(x)=\phi * \stackrel{\vee}{\phi}(x), x \in \mathbb{R}$, where $\stackrel{\vee}{\phi}(x):=\phi(-x)$ and $*$ stands for convolution. Clearly $f(x)=f(-x)$ and $f$ is symmetric. Let $\Phi(i \lambda)=\mathbb{E}\left(e^{i \lambda X_{1}}\right)$ be the common characteristic function of both $X_{1}$ and $X_{2}$. Then $|\Phi(i \lambda)|^{2}$ is the (real) Fourier transform of $f(x)$ or the characteristic function of $X$. Clearly $g(\lambda):=\frac{1}{2 \pi}|\Phi(i \lambda)|^{2} \geq 0$ for almost all $\lambda$ and since $f$ is continuous and integrable, 
by Bochner theorem, $f$ is definite non-negative in that, for all integer $n$, all real numbers $\mathbf{x}:=\left(x_{m}, m=1, . ., n\right)$ and all $\mathbf{z} \in \mathbb{C}^{n}: \mathbf{z}^{\prime}\left[f\left(x_{m}-x_{m^{\prime}}\right)\right] \mathbf{z} \geq 0$. In this interpretation, the function $g(\lambda)$ should be regarded as the integrable spectral density associated to the correlation function $f$. Here, $\left[f\left(x_{m}-x_{m^{\prime}}\right)\right]$ is the $n \times n$ square matrix whose $\left(m, m^{\prime}\right)$ entry is $f\left(x_{m}-x_{m^{\prime}}\right)$. Assuming $W=\left[W_{m, m^{\prime}}\right]$ and $W_{m, m^{\prime}}=f\left(x_{m}-x_{m^{\prime}}\right)$, then the weight matrix is doubly non-negative. It should be emphasized that strictly speaking, $W=W(\mathbf{x})$, now is a function of $\mathbf{x}$. Note also that this construction yields a correlation function which is also a probability density function so that total mass of $f$ is 1 , which is purely arbitrary.

Let $\psi(x) \geq 0$ be any non-negative function on $\mathbb{R}$. Then, with $W_{m, m^{\prime}}=$ $\psi\left(x_{m}\right) f\left(x_{m}-x_{m^{\prime}}\right) \psi\left(x_{m^{\prime}}\right), W:=\left[W_{m, m^{\prime}}\right]$ is diagonally congruent to the latter and so is also doubly non-negative. Clearly, its entries are indeed non negative and definite positiveness is preserved under congruence. If $\psi(x)=C>0$, this constant can be used to readjust the mass of $f$ if needed.

\section{Remarks.}

(i) Let $P$ be the permutation matrix which maps the indices of $\left(x_{m}, m=1, . ., n\right)$ into the ones of $-\infty<x_{1} \leq . . \leq x_{n}<\infty$ where now the $x_{m}$ are ordered on the real line. By considering instead the novel congruent weight matrix $P^{\prime} W P$, we can assume, without loss of generality, that $W_{m, m^{\prime}}=\psi\left(x_{m}\right) f\left(x_{m}-x_{m^{\prime}}\right) \psi\left(x_{m^{\prime}}\right)$ where the $x_{m}$ are ordered. Note that in this construction, a particle is attached to box number $m$ if and only if it stands at $m$-th position $x_{m}$ on the real line. In this context, $H_{m, m^{\prime}}:=-\log W_{m, m^{\prime}}$ is now the energy required to move a particle from site $x_{m}$ to site $x_{m^{\prime}}$.

(ii) let $w_{x}$ be a (stationary) white noise $(\delta$-correlated) process, indexed by $x \in \mathbb{R}$. Let $z_{x}:=(\phi * w)_{x}$ where $\phi$ is as above. Then $z_{x}, x \in \mathbb{R}$, is a stationary process whose correlation function is $\operatorname{Cov}\left[z_{x^{\prime}} z_{x^{\prime}+x}\right]=f(x)=\phi * \stackrel{\vee}{\phi}(x)$.

(iii) Assume both $\left(X_{1}, X_{2}\right)$ have common law supported by $(0, \infty)$ in the above construction. Then, it can be checked that $f(x)=h(|x|)$ where $h(z)=$ $\int_{0}^{\infty} \phi(y+z) \phi(y) d y, z>0$.

(iv) Assume both $\left(X_{1}, X_{2}\right)$ have symmetric common law supported by $\mathbb{R}$. Then $f(x)=\phi^{* 2}$ and $\mathbb{E}\left(e^{i \lambda X_{1}}\right)$ is real. A given $f$ belongs to this class if it is 2 -divisible on $\mathbb{R}$. Note that $f(x)=h(|x|)$ where $h(z)=\int_{\mathbb{R}} \phi(z-y) \phi(y) d y$, $z>0$.

$(v)$ Exploiting this Fourier isomorphism on integrable positive functions, reversing the role played by 'space' $x$ and wave number $\lambda$, we conclude that $f(x)=\frac{1}{2 \pi}|\Phi(i x)|^{2}$ is a correlation function whose associated spectral measure is $g(\lambda)=\phi * \phi(\lambda), \lambda \in \mathbb{R}$. $\diamond$

Examples. ( $i)$ Let $\phi(x)=e^{-x} \mathbf{1}(x>0)$. Then, $f(x)=e^{-|x|} / 2, x \in \mathbb{R}$, is a continuous integrable correlation function with spectral density $g(\lambda)=$ 
$1 /\left[\pi\left(1+\lambda^{2}\right)\right]$. For all $n$ and $-\infty<x_{1} \leq . . \leq x_{n}<\infty$, the matrix $W=$ $\left[e^{-\left|x_{m}-x_{m^{\prime}}\right|}\right]$ has the required properties. Let $\psi(x)=e^{x}$. Then, with $W_{m, m^{\prime}}=$ $e^{x_{m}} e^{-\left|x_{m}-x_{m^{\prime}}\right|} e^{x_{m^{\prime}}}=\min \left(e^{2 x_{m}}, e^{2 x_{m^{\prime}}}\right), W:=\left[W_{m, m^{\prime}}\right]$ also has the required properties. Specifying $x_{m}$ to $x_{m}=(\log m) / 2$, the matrix with entries $\min \left(m, m^{\prime}\right)$ has the required properties. Putting $t_{m}=e^{x_{m}}, W:=\left[\min \left(t_{m}, t_{m^{\prime}}\right)\right]$ has the required property where we recognize the correlation kernel of standard Brownian motion on $\mathbb{R}_{+}$.

(ii) Let $\phi(x)=\frac{1}{\sqrt{2 \pi}} e^{-x^{2} / 2}, x \in \mathbb{R}$.

Then $g(\lambda)=\frac{1}{2 \pi} e^{-\lambda^{2}}$, showing that $f(x)=\phi^{* 2}(x)=\int_{\mathbb{R}} e^{-i \lambda x} g(\lambda) d \lambda=$ $\frac{1}{2 \sqrt{\pi}} e^{-x^{2} / 4}, x \in \mathbb{R}$.

(iii) Assume $\phi(x)=\exp \left[-\left(x+e^{-x}\right)\right]$ so that $X_{1}$ has Gumbel density on $\mathbb{R}$. One can check that $f(x)=1 /[2(1+\cosh (x))]$. We have $\Phi(i \lambda)=\Gamma(1-i \lambda)$ and $g(\lambda):=|\Gamma(1-i \lambda)|^{2} /(2 \pi)=\lambda / 2 \sinh (\lambda \pi)$.

More generally, let $\beta>0$ and assume now $\phi(x)=\exp \left[-\left(\beta x+e^{-x}\right)\right]$ on $\mathbb{R}$. Then, with $B(.,$.$) the Euler beta function, f(x)=[1 / 2(\cosh (x / 2))]^{2 \beta} / B(\beta, \beta)$. We have $\Phi(i \lambda)=\Gamma(\beta-i \lambda) / \Gamma(\beta)$ and $g(\lambda):=|\Gamma(\beta-i \lambda)|^{2} /(2 \pi)$.

Specifying $\beta=1 / 2$, we obtain

$$
f(x)=1 /(2 \pi \cosh (x / 2)) \text { and } g(\lambda)=1 /[2 \pi \cosh (\pi \lambda)] .
$$

This shows that the pair $f(x)=1 / \cosh (x)$ and $g(\lambda)=1 / \cosh (\pi \lambda / 2)$ is admissible. Thus $W_{m, m^{\prime}}=1 / \cosh \left(x_{m}-x_{m^{\prime}}\right)$ but also

$$
W_{m, m^{\prime}}=e^{x_{m}} e^{x_{m^{\prime}}} /\left[2 \cosh \left(x_{m}-x_{m^{\prime}}\right)\right]=1 /\left(e^{-2 x_{m}}+e^{-2 x_{m^{\prime}}}\right)
$$

is doubly non-negative. Assuming a lattice case $x_{m}=(-\log m) / 2$, the Cauchy matrix with entries $1 /\left(m+m^{\prime}\right)$ has the required properties.

(iv) Assume $f(x)=\frac{1}{2 \Gamma(1+1 / \gamma)} e^{-|x|^{\gamma}}, x \in \mathbb{R}, \gamma \in(0,1]$. The above construction could apply if, in particular, one could prove that there is a $\phi$ supported by $(0, \infty)$ such that $\frac{1}{\Gamma(1+1 / \gamma)} e^{-z^{\gamma}}=\int_{0}^{\infty} \phi(y+z) \phi(y) d y, z>0$. But, unless $\gamma=1$, this is not the case. In fact, in this case, there is a symmetric $\phi$ such that $f(x) \propto e^{-|x|^{\gamma}}=\phi^{* 2}$. $\phi$ is characterized by $2 \int_{0}^{\infty} \cos (\lambda x) \phi(x) d x=[2 \pi g(\lambda)]^{1 / 2}$ where $g(\lambda)$ is a symmetric stable $(\gamma)$ density (see Feller, [10], page 583, for an expression). Indeed, $f(x)$ is a symmetric distribution whose density restricted to $(0, \infty)$, namely $f_{+}(x)=\frac{1}{\Gamma(1+1 / \gamma)} e^{-x^{\gamma}}, x \in \mathbb{R}_{+}, \gamma \in(0,1)$, is completely monotone. By theorem 10.1 page 202 of Steutel, van Harn, [20], $f(x)$ is infinitely divisible so that, for $k$ integer, there is a probability density $\phi_{k}$ on $\mathbb{R}$ such that $f=\phi_{k}^{* k}$. The above claim follows from this with $k=2$.

However, it is not necessary to prove this to conclude that $f(x)$ (even when $\gamma \in(0,2])$ is indeed a correlation function because $g(\lambda):=\frac{1}{2 \pi} \int_{\mathbb{R}} e^{i \lambda x} f(x) d x$ is a stable (strictly positive) density with shape parameter $\gamma$ and so is $\geq 0$ for almost all $\lambda$. 
$(v)$ Assume $\phi(x)=\mathbf{1}(x \in[0,1])$. Then, $f(x)=(1-|x|) \mathbf{1}(x \in[-1,1])$ and $g(\lambda)=\frac{1}{2 \pi}\left(\frac{\sin \lambda / 2}{\lambda / 2}\right)^{2}$ is an admissible pair. Note that $\mathbf{1}(x \in[0,1])$ is not itself a correlation function as its Fourier transform, which is $\frac{1}{\pi} \frac{\sin \lambda}{\lambda}$ is not $\geq 0$.

The set of doubly non-negative correlation functions forms a closed cone as this property is preserved under convex linear combinations and point-wise products and if a sequence $\left(f_{k} ; k \geq 1\right)$ of such correlation functions converges, then the limit remains doubly non-negative.

If $f_{1}$ and $f_{2}$ are 2 doubly non-negative correlation functions with associated weight matrices $W_{i}=\left[f_{i}\left(x_{m}-x_{m^{\prime}}\right)\right], i=1,2$, then the weight matrix $W$ associated to $f=f_{1} \cdot f_{2}$ is $W=W_{1} \circ W_{2}$ where $\circ$ stands for Hadamard (or Schur) entry-wise product. The Hadamard product of doubly non-negative matrices indeed is a doubly non-negative matrix.

Fix $1 \leq m_{1}<. .<m_{p} \leq n$ a subsequence of length $p$ from $\{1, . ., n\}$. Considering the principal sub-matrices $W\left(\left\{m_{1}, . ., m_{p}\right\}\right)$ of $W$, we get a submatrix of the same type as the original one. This is useful in the computation of $\left|W\left(\left\{m_{1}, . ., m_{p}\right\}\right)\right|$ since this can be read from the one of $|W|$. For instance, if $W=\left[\left(e^{x_{m}}+e^{x_{m^{\prime}}}\right)^{-1}\right]$, it has been known since Cauchy that

$$
|W|=\frac{\prod_{1 \leq m<m^{\prime} \leq n}\left(e^{x_{m^{\prime}}}-e^{x_{m}}\right)^{2}}{\prod_{1 \leq m<m^{\prime} \leq n}\left(e^{x_{m^{\prime}}}+e^{x_{m}}\right)} .
$$

From this, we immediately get

$$
\left|W\left(\left\{m_{1}, . ., m_{p}\right\}\right)\right|=\frac{\prod_{1 \leq q<q^{\prime} \leq p}\left(e^{x_{m_{q^{\prime}}}}-e^{x_{m_{q}}}\right)^{2}}{\prod_{1 \leq q<q^{\prime} \leq p}\left(e^{x_{m_{q^{\prime}}}}+e^{x_{m_{q}}}\right)} .
$$

\section{Infinitely divisible weight matrices.}

We shall briefly discuss here conditions under which raising $W$ to the power $\beta$ (in some sense) gives birth to a family of doubly non-negative matrices for all $\beta>0$.

- Let $H$ be a $n \times n$ symmetric matrix. With $\beta>0$, consider the weight matrix $W_{\beta}:=e^{-\beta H}$. For all $\beta>0$, this weight matrix is definite non-negative (eigenvalues are real and non-negative) but, unless some extraordinary circumstances, its off-diagonal entries have no reason to be non-negative, even if $W=e^{-H}$ were chosen so as to have itself non-negative entries. A question could be: which are the doubly non-negative weight matrices $W$ whose $\beta$-powers remain doubly non-negative for all $\beta>0$ ? Preserving positive definiteness and entry-wise positivity of (standard) $\beta$-powers together is a very rare combination and very 
much basis-dependent. We now turn to a related question which is more meaningful in our context.

- Let $W$ be a doubly non-negative weight matrix. With $\beta>0$, let $W^{\circ \beta}$ be the Hadamard $\beta$-power of $W$, with entries $\left(W^{\circ \beta}\right)_{m, m^{\prime}}:=W_{m, m^{\prime}}^{\beta}$. This new matrix has non-negative entries and, in any case, when $\beta \geq \beta_{c}:=n-2$ it remains definite non-negative (see Fitzgerald and Horn, [11]). However (when $n \geq 3$ ), only under some peculiar circumstances is it still a non-negative definite matrix for all $\beta>0$ (although by Schur theorem it always is when $\beta$ is an integer). Doubly non-negative matrices whose Hadamard $\beta$-powers remain doubly nonnegative for all $\beta>0$ are called infinitely divisible (ID) matrices (see Bhatia, [3]). The Schur product of infinitely divisible matrices yields a novel infinitely divisible matrix. Examples of infinitely divisible matrices are

$$
\begin{gathered}
W_{m, m^{\prime}}=\left(e^{x_{m}}+e^{x_{m^{\prime}}}\right)^{-1}, \Gamma\left(e^{x_{m}}+e^{x_{m^{\prime}}}+1\right) /\left[\Gamma\left(e^{x_{m}}+1\right) \Gamma\left(e^{x_{m^{\prime}}}+1\right)\right], \\
\max \left(e^{x_{m}}, e^{x_{m^{\prime}}}\right)^{-1}=\min \left(e^{-x_{m}}, e^{-x_{m^{\prime}}}\right), \cosh \left(\frac{1}{2}\left(x_{m}-x_{m^{\prime}}\right)\right)^{-1}, \\
\exp \left[-\left|x_{m}-x_{m^{\prime}}\right|\right] \text { and more generally } \exp \left[-\left|x_{m}-x_{m^{\prime}}\right|^{\gamma}\right] \text { for } \gamma \in(0,2] .
\end{gathered}
$$

where $-\infty<x_{1} \leq x_{2} \leq . . \leq x_{n}<\infty$

In some (stationary) cases, the correlation kernel $W_{m, m^{\prime}}=W\left(x_{m}, x_{m^{\prime}}\right)$ only depends on the difference of $\left(x_{m}, x_{m^{\prime}}\right)$.

\section{Remark.}

In the above language to construct doubly non-negative weight matrices $W$ from positive correlation functions $f$, it can be checked directly that $W$ is infinitely divisible if and only if $f(x)^{\beta}$ remains a correlation function for all $\beta>0$ which clearly is the case for the examples displayed above. If the correlation function $f$ fulfills this property, then, considering its spectral density $g(\lambda)$, for each integer $k$, there is a spectral measure $g_{k}(\lambda)$ such that $g=g_{k}^{* k}$. In this context, infinite divisibility is the eventual property of the spectral density. $\diamond$

For such infinitely-divisible doubly non-negative matrices (with strictly positive entries), the Hadamard logarithm of $W$, defined by

$$
H:=\left[H_{m, m^{\prime}}\right] \text { and } H_{m, m^{\prime}}=-\log W_{m, m^{\prime}},
$$

satisfies $\mathbf{z}^{\prime} H \mathbf{z} \leq 0$ for all $\mathbf{z} \in \mathbb{C}^{n}$ such that $\sum_{m=1}^{n} z_{m}=0$; the 'energy' matrix $H$ is said to be conditionally definite non-positive. The converse is also true: conditional definite non-positivity of $H$ implies infinite divisibility of $W=e^{-\circ H}$ (see Loewner, [18]).

Further, $H$ is conditionally definite non-positive if and only if the $(n-1) \times$ $(n-1)$ matrix $\Delta$ with entries

$$
\Delta_{m, m^{\prime}}=H_{m, m^{\prime}}+H_{m+1, m^{\prime}+1}-H_{m, m^{\prime}+1}-H_{m+1, m^{\prime}}, m, m^{\prime} \in\{1, . ., n-1\}
$$


is definite non-positive $\left(\mathbf{z}^{\prime} H \mathbf{z} \leq 0\right.$ for all $\left.\mathbf{z} \in \mathbb{C}^{n}\right)$.

\section{Example.}

The matrix $W_{m, m^{\prime}}=\exp \left[\left|x_{m}\right|^{\gamma}+\left|x_{m^{\prime}}\right|^{\gamma}-\left|x_{m}-x_{m^{\prime}}\right|^{\gamma}\right]$ is ID because it is congruent to an ID matrix when $\gamma \in(0,2]$. Therefore $-H_{m, m^{\prime}}:=\log W_{m, m^{\prime}}=$ $\left|x_{m}\right|^{\gamma}+\left|x_{m^{\prime}}\right|^{\gamma}-\left|x_{m}-x_{m^{\prime}}\right|^{\gamma}$ is conditionally definite non-negative. In the latter expression, we can recognize the correlation kernel of fractional Brownian motion with Hurst exponent $\gamma / 2$.

A block-diagonal matrix whose Jordan blocks are individually infinitely divisible is globally infinitely divisible. So is a matrix which be brought into this form after a simultaneous permutation of its rows and columns.

Assume particles can only be placed in boxes in the positions $-\infty<x_{1} \leq$ $x_{2} \leq . . \leq x_{n}<\infty$ on the real line. In this setting, the box number of a particle is the index of its position on $\mathbb{R}$ and the model is spatially extended. Let $W^{\circ \beta}$ with $W=e^{-\circ H}$ infinitely divisible where $H_{m, m^{\prime}}=H\left(x_{m}, x_{m^{\prime}}\right)$ are, say, as in the previous examples. For such matrices $W$, for all $\alpha \in(0, \infty], \beta>0$

$$
\mathbb{E}_{\alpha}\left(\prod_{m=1}^{n} u_{m}^{K_{n, z}(m)}\right)=\left(\frac{\left|I-z_{\alpha} W^{\circ \beta}\right|}{\left|I-z_{\alpha} U W^{\circ \beta}\right|}\right)^{\alpha}
$$

is the probability generating function of a well-defined infinitely divisible random vector corresponding to box occupancies associated to $W^{\circ \beta}$. When $\alpha=1$ (BoseEinstein statistics), it addresses the problem of computing the grand-canonical weight of the configurations obtained by rearranging an average number $\kappa=$ $\kappa(z)$ of multi-type particles when the weight associated to transition $m$ to $m^{\prime}$ at 'temperature' $1 / \beta>0$ is $e^{-\beta H_{m, m^{\prime}}}$. The probability of these configurations follows next upon normalizing. Since for all $\beta>0, W^{\circ \beta}$ has all its minors non-negative, it is also the probability generating function of a well-defined generalized multinomial random variable when $\alpha \in\{. .,-2,-1\}$. We note that, since $W^{\circ \beta}$ is definite non-negative

$$
\left|I-z_{\alpha} U W^{\circ \beta}\right|^{-\alpha}=\left|I-z_{\alpha} U^{1 / 2} W^{\circ \beta} U^{1 / 2}\right|^{-\alpha}=\prod_{m=1}^{n}\left(1-z_{\alpha} \lambda_{m, \beta}(\mathbf{u})\right)^{-\alpha}
$$

where $\lambda_{m, \beta}(\mathbf{u}), m=1, . ., n$ are the real non-negative eigenvalues of $U^{1 / 2} W^{\circ \beta} U^{1 / 2}$ which is congruent to $W^{\circ \beta}$ and definite non-negative for all $\mathbf{u} \in[0,1]^{n}$. Therefore, with $\lambda_{m, \beta}:=\lambda_{m, \beta}(\mathbf{1})$ the eigenvalues of $W^{\circ \beta}$

$$
\mathbb{E}_{\alpha}\left(\prod_{m=1}^{n} u_{m}^{K_{n, z}(m)}\right)=\left(\frac{\left|I-z_{\alpha} W^{\circ \beta}\right|}{\left|I-z_{\alpha} U W^{\circ \beta}\right|}\right)^{\alpha}=\prod_{m=1}^{n}\left(\frac{1-z_{\alpha} \lambda_{m, \beta}}{1-z_{\alpha} \lambda_{m, \beta}(\mathbf{u})}\right)^{-\alpha} .
$$

This shows that the probability generating function of $\mathbf{K}_{n, z}$ under $\mathbb{P}_{\alpha}$ can be factorized. As a very particular (interaction-free) example, if $0<x_{1} \leq x_{2} \leq . . \leq$ 
$x_{n}<1$ and if $W=\operatorname{diag}\left(x_{1}, . ., x_{n}\right)$, then $H=\operatorname{diag}\left(\epsilon_{1}, . ., \epsilon_{n}\right)$ with $\epsilon_{m}=-\log x_{m}$, $0<\epsilon_{n} \leq . . \leq \epsilon_{1}$ and

$$
\mathbb{E}_{\alpha}\left(\prod_{m=1}^{n} u_{m}^{K_{n, z}(m)}\right)=\left(\frac{\left|I-z_{\alpha} W^{\circ \beta}\right|}{\left|I-z_{\alpha} U W^{\circ \beta}\right|}\right)^{\alpha}=\prod_{m=1}^{n}\left(\frac{1-z_{\alpha} e^{-\beta \epsilon_{m}}}{1-z_{\alpha} u_{m} e^{-\beta \epsilon_{m}}}\right)^{\alpha}
$$

with independent negative binomial factors. In particular, if $\alpha>0$

$$
\mathbb{P}_{\alpha}\left(\mathbf{K}_{n, z}=\mathbf{k}_{n}\right)=\prod_{m=1}^{n}\left(1-z_{\alpha} e^{-\beta \epsilon_{m}}\right)^{\alpha} \cdot z_{\alpha}^{\left|\mathbf{k}_{n}\right|} \prod_{m=1}^{n} \frac{(\alpha)_{k_{m}}}{k_{m} !} e^{-\beta k_{m} \epsilon_{m}}, \mathbf{k}_{n} \in \mathbb{N}^{n}
$$

which favors states with lower energy. This model is familiar in occupancy problems of discretized energy levels.

Acknowledgments: I should like to thank Professor David Vere-Jones, from the University of Wellington, New-Zealand, for sending me some of the relevant literature. This largely motivated the present work. 


\section{References}

[1] Aringazin A.K.; Mazhitov, M.I. Combinatorial interpretation of HaldaneWu fractional exclusion statistics. Phys. Rev. E 66, 026116, 2002.

[2] Bapat, R. B. Permanents in probability and statistics. Linear Algebra Appl. 127, 3-25, 1990.

[3] Bhatia, R. Infinitely divisible matrices. Amer. Math. Monthly 113, No. 3, 221-235, 2006.

[4] Bialas, P.; Burda, Z.; Johnston, D. Condensation in the Backgammon model. Nucl. Phys. B 493, 505-516, 1997.

[5] Cartier, P.; Foata, D. Problèmes combinatoires de commutation et réarrangements. Lecture Notes in Mathematics, 85, Berlin, Springer-Verlag, 1969.

[6] Charalambides, C. A. Combinatorial methods in discrete distributions. Wiley Series in Probability and Statistics. Wiley-Interscience [John Wiley \& Sons], Hoboken, NJ, 2005.

[7] Chu, W. Determinant, permanent, and MacMahon's master theorem. Linear Algebra Appl. 255, 171-183, 1997.

[8] Comtet, L. Analyse combinatoire. Tomes 1 et 2. Presses Universitaires de France, Paris, 1970.

[9] Diaconis, P.; Graham, R.; Holmes, S.P. Statistical problems involving permutations with restricted positions. State of the art in probability and statistics (Leiden, 1999), 195-222, IMS Lecture Notes Monogr. Ser., 36, Inst. Math. Statist., Beachwood, OH, 2001.

[10] Feller W. An introduction to probability theory and its applications, 2, Wiley, New York, 1971.

[11] FitzGerald, C. H.; Horn, R. A. On fractional Hadamard powers of positive definite matrices. J. Math. Anal. Appl. 61, No. 3, 633-642, 1977.

[12] Foata, D.; Zeilberger, D. Laguerre polynomials, weighted derangements, and positivity. SIAM J. Discrete Math. 1, No. 4, 425-433, 1988.

[13] Gillis, J. The statistics of derangements - A survey. J. Stat. Phys., 58, Nos 3-4, 1990.

[14] Greenberg, O.W.; Greenberger, D.M.; Greenbergest, T.V. (Para)bosons, (para)fermions, quons and other beasts in the menagerie of particle statistics. hep-ph/9306225, 1993. 
[15] Huillet, T. Occupancies within zeta urns revisited. available at: http://hal.ccsd.cnrs.fr/ccsd-00096631, 2006.

[16] Johnson, N. L.; Kotz, S. Urn models and their application. An approach to modern discrete probability theory. Wiley Series in Probability and Mathematical Statistics. John Wiley \& Sons, New York-London-Sydney, xiii+402 pp., 1977.

[17] Kolchin, V. F. Random mappings. Translated from the Russian. With a foreword by S. R. S. Varadhan. Translation Series in Mathematics and Engineering. Optimization Software, Inc., Publications Division, New York, 1986.

[18] Loewner, C. On schlicht-monotonic functions of higher order. J. Math. Anal. Appl. 14, 320-325, 1966.

[19] Mehta, M.L. Matrix theory. Selected topics and useful results. Hindustan Publishing Corporation. 1989.

[20] Steutel, F. W.; van Harn, K. Infinite divisibility of probability distributions on the real line. Monographs and Textbooks in Pure and Applied Mathematics, 259. Marcel Dekker, Inc., New York, 2004.

[21] Vere-Jones, D. Alpha-permanents and their applications to multivariate gamma, negative binomial and ordinary binomial distributions. New Zealand J. Math. 26, No. 1, 1997.

[22] Vere-Jones, D. Permanents, determinants, bosons and fermions. New Zealand Math. Soc. Newslett. No. 29, 18-23, 1983. 